\title{
Chemical Shift Tensors - Why Should We Care?
}

\author{
Christopher P. Gordon§ and Christophe Copéret ${ }^{\star}$ \\ §SCS-Metrohm Award for best oral presentation in Inorganic \& Coordination Chemistry
}

\begin{abstract}
Chemical shift tensors give valuable insights into the nature and the relative energy of frontier orbitals and their analysis allows for rationalizing the reactivities of molecules. In this article, we point out the principles that allow for the analysis of chemical shift. Through selected, illustrative examples we show how one can relate chemical shift to molecular electronic structure and thus to reactivity.
\end{abstract}

Keywords: Chemical shift tensor - Frontier molecular orbitals · Natural chemical shift analysis · NMR spectroscopy $\cdot$ Reactivity descriptor

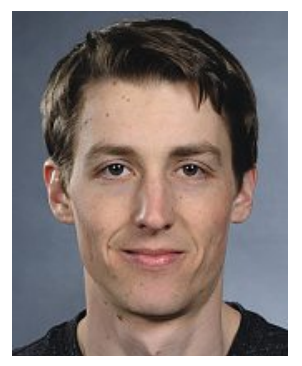

Christopher P. Gordon was awarded a Bachelor's degree in Chemistry at ETH Zürich in 2015. He completed his Master's degree at ETH Zürich under the Excellence Scholarship and Opportunity Programme in 2017 and was awarded the ETH Medal for his Master's thesis. He is currently pursuing a PhD degree in the group of Prof. Christophe Copéret at ETH Zürich, focusing on analysis of chemical shift as a descriptor to understand reactive intermediates in catalysis.

\section{Introduction}

NMR spectroscopy is one of the most potent and widely used tools to investigate the structure and dynamics of molecules and materials. ${ }^{[1]}$ One of the most compelling properties of NMR is its sensitivity to the local electronic environment of nuclei: the resonance frequency of a nucleus is not only determined by the type of nucleus $\left(\right.$ e.g. ${ }^{1} \mathrm{H},{ }^{11} \mathrm{~B},{ }^{13} \mathrm{C},{ }^{15} \mathrm{~N},{ }^{17} \mathrm{O}$, etc. $)$ but also by its direct chemical environment. ${ }^{[2]}$ This feature of NMR gives rise to the socalled chemical shift and allows to distinguish nuclei of the same type in different chemical environments $\left(e . g .{ }^{13} \mathrm{C}\right.$ in alkyl groups, in olefinic positions, or in carbonyl positions), making NMR one of the most powerful tools to characterize molecules and materials in the laboratory. ${ }^{[3]}$

The origin of chemical shift is a local strengthening or weakening of the external magnetic field due to the molecular electronic structure. We generally accept that $\mathrm{sp}^{2}$ carbon-atoms in olefins are more deshielded than a $\mathrm{CH}_{3}$ group ${ }^{[4]}$ or that many transition-metal alkyl species show highly deshielded carbon chemical shifts in sharp contrast to alkyl-lithium or Grignard reagents. ${ }^{[3 a]}$ Besides being a powerful signature helping to determine molecular structures, chemical shift contains more information. In this article we describe how the chemical shift of an atom relates to frontier molecular orbitals, and how we can use chemical shift as a powerful descriptor to delineate the local electronic environment of nuclei and understand the reactivity of molecules.

\footnotetext{
${ }^{*}$ Correspondence: Prof. C. Copéret

\section{Chemical Shift and Chemical Shift Tensors}

When we measure NMR spectra in solution, we typically obtain one chemical shift value for each magnetically inequivalent nucleus. This value is the so-called isotropic chemical shift value $\left(\delta_{\text {iso }}\right)$. However, chemical shift is an anisotropic property the resonance frequency (and hence the chemical shift) depends on the orientation of the molecule with respect to the external magnetic field. In solution NMR, this orientation dependence is averaged out by the fast tumbling of the molecules. However, when measured in the solid state, molecular tumbling is supressed and instead of isotropic chemical shift values one can see the so-called powder pattern that arises from the orientation dependence of the chemical shift with respect to the external magnetic field; it also provides access to the principal components of the chemical shift tensor as illustrated for ethylene in Fig. 1.

The chemical shift tensor can be described by its three principal components, which are defined as $\delta_{11} \geq \delta_{22} \geq \delta_{33}$ and whose average corresponds to $\delta_{\text {iso }}$, observed in solution. These three components are orthogonal to each other and their magnitude and orientation can provide significant insight into the local electronic structure of molecules and help identifying the nature of frontier molecular orbitals (vide infra). ${ }^{[4,5]}$

The chemical shift values $(\delta)$ are reported with respect to a reference compound, whose chemical shift is arbitrarily set to 0 ppm. For ${ }^{13} \mathrm{C}$ NMR spectra, tetramethylsilane - TMS - is typically used as a reference and set to $0 \mathrm{ppm}$. The related chemical shielding $(\sigma)$, an intrinsic property of the nuclei in molecules, describes the shielding or deshielding of a nucleus with respect to the bare nucleus whose shielding is set to $0 \mathrm{ppm}$. While experimentally only the chemical shift $(\delta)$ can be measured, in computational approaches (e.g. DFT calculations) the chemical shielding $(\sigma)$ is not only calculated, but can also be analysed and related to detailed electronic structure.

\subsection{Relating Shielding and Deshielding to Electronic Structure}

Each principal component of the chemical shielding tensor $\left(\sigma_{\mathrm{ii}}\right)$ can be decomposed into diamagnetic $\left(\sigma_{\mathrm{dia}}\right)$ and paramagnetic $\left(\sigma_{\text {para }}\right)$ terms (Eqn. (1)). The diamagnetic shielding arises mostly from core orbitals and causes a rather isotropic shielding of the nucleus. Since core orbitals are similar among nuclei in various chemical environments in most cases, the diamagnetic shielding is usually rather independent of the local molecular structure. On the 


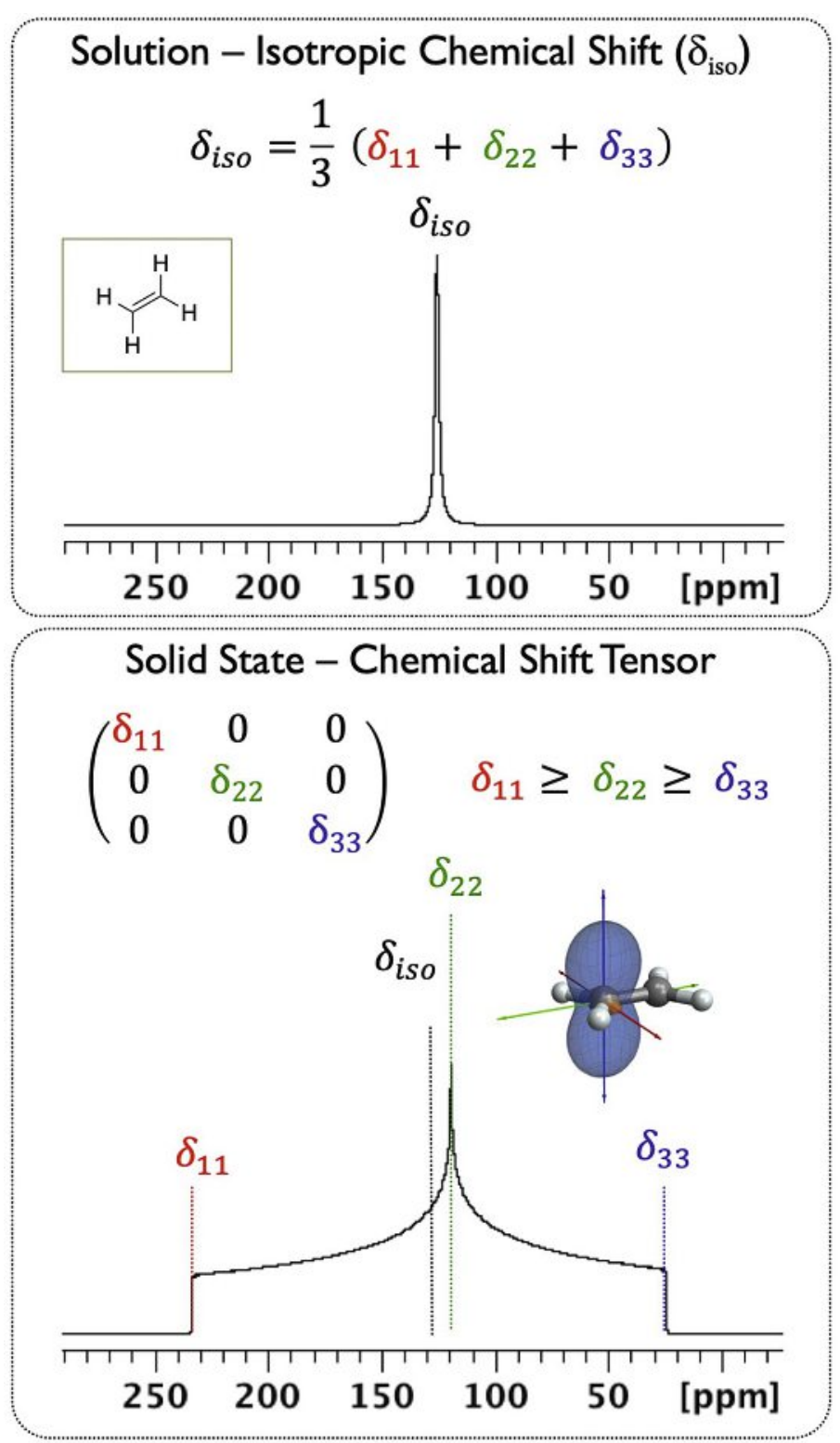

Fig. 1. Simulated NMR spectra of ethylene in solution and in the solid state. Due to molecular tumbling, in solution only the isotropic value of the chemical shift tensor is observed, whereas in the solid state a powder pattern is obtained, from which all three principal components of the chemical shift tensor can be extracted.

contrary, the paramagnetic shielding arises from the interaction of the molecular electronic ground state with excited states by a coupling through the angular momentum operator $\hat{L}$ (Eqn. (2)), typically leading to deshielding. This deshielding is particularly pronounced for the coupling of energetically low-lying excited states and a high-lying ground state, corresponding to frontier molecular orbitals of appropriate symmetry to allow magnetically induced coupling. In an orbital view, a large paramagnetic deshielding of a nucleus is expected along a direction $i$, whenever a high-lying occupied orbital on this nucleus can be superimposed on a low-lying vacant orbital on the same nucleus by a rotation by $90^{\circ}$ (in the case of p-orbitals, which are usually dominant for main group nuclei) along the $i$-axis (as illustrated for ethylene below - Fig. 2). ${ }^{[5]}$

$\sigma_{i i}=\sigma_{\mathrm{ii}, \mathrm{dia}}+\sigma_{\mathrm{ii}, \mathrm{para}+\mathrm{So}}$

$\sigma_{i i, \text { para }} \Leftrightarrow-\frac{\left\langle\Psi_{\text {occ }}\left|\hat{L}_{i}\right| \Psi_{\text {vac }}\right\rangle\left\langle\Psi_{\text {vac }}\left|\hat{L}_{i} / r^{3}\right| \Psi_{\text {occ }}\right\rangle}{\Delta E_{\text {vac }- \text { occ }}}$

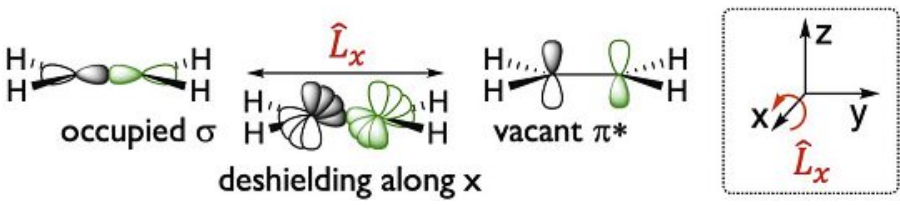

Fig. 2. Coupling of high-lying occupied and vacant orbitals by the angular momentum operator $\hat{L}_{x}$, leading to deshielding along the $x$-axis.

The chemical shielding tensor $(\sigma)$ can be calculated by theoretical methods, for instance by DFT calculations. These calculations allow for determining the value and the orientation of the principal components of the shielding tensor and for identifying the contributions of specific occupied orbitals. A particularly useful approach is the so called Natural Chemical Shielding (NCS) analysis, in which the occupied orbitals with relevance for the shielding/deshielding of a given nucleus are expressed in terms of Natural Localized Molecular Orbitals (NLMOs). ${ }^{[6]}$ Since NLMOs correspond to bonds and lone pairs this analysis of the chemical shielding allows for a very intuitive view of the origin of a specific chemical shielding.

\subsection{Methodology}

The chemical shielding tensors shown in this contribution were obtained by first optimizing the molecular structures with the gaussian09 program $^{[7]}$ (using a PBE0 functional[ ${ }^{8]}$ in combination with pcseg- 2 basis sets of triple- $\zeta$ quality ${ }^{[9]}$ and, if applicable, quasi-relativistic effective core potentials of the Stuttgart-group and the associated basis sets on metal atoms $\left.{ }^{[10]}\right)$. NMR calculations were performed using ADF 2014 within the GIAO framework, ${ }^{[11]}$ with a PBE0 functional[8] and Slater-type basis sets of triple- $\zeta$ quality. For the NMR calculations, relativistic effects are treated by the 2 component zeroth order regular approximation (ZORA). ${ }^{[2]}$ The obtained NMR shielding tensors are analysed using scalar relativistic natural localized molecular orbitals (NLMOs), obtained with the NBO 6.0 program. ${ }^{[13]}$ Moreover, chemical shift tensors can be obtained experimentally through solid-state NMR measurements that can be performed on powdered and microcrystalline solids as well as frozen solutions, which are packed into an NMR rotor and measured by magic angle spinning solid state NMR. The spinning rate is chosen so as to give enough spinning side-bands to allow for extraction of the principal components of the chemical shift tensor by fitting of the obtained spectrum. These experimentally determined chemical shift tensors are important to benchmark DFT calculations.

\section{Shielding Tensors of Representative Molecules and their Connection to Electronic Structure}

\subsection{Ethylene}

The calculated shielding tensor of ethylene is shown in Fig. 3. As expected for olefins, the isotropic chemical shift $\left(\delta_{\text {iso }}=132 \mathrm{ppm}\right)$ is rather deshielded by comparison with $\mathrm{sp}^{3}$-carbon atoms (e.g.7 ppm in ethane). A closer analysis of the individual components of the chemical shift tensor reveals that this large deshielding of ethylene is mainly due to a highly deshielded $\delta_{11}$ component $(258$ $\mathrm{ppm})$, while the $\delta_{33}$ component (14 ppm) is similar to what is found for $\mathrm{sp}^{3}$ carbon atoms. This most deshielded component $\left(\delta_{11} / \sigma_{11}\right)$ is oriented perpendicular to the $\sigma(\mathrm{C}=\mathrm{C})$ and the $\pi(\mathrm{C}=\mathrm{C})$ bonds. The intermediate component $\left(\delta_{22} / \sigma_{22}\right)$ is oriented along the $\sigma(C=C)$ bond, while the most shielded component $\left(\delta_{33} / \sigma_{33}\right)$ is perpendicular to both $\delta_{11} / \sigma_{11}$ and $\delta_{22} / \sigma_{22}$. An orbital decomposition (NCS analysis) of the individual components of the shielding tensor $\left(\sigma_{11}, \sigma_{22}\right.$, and $\sigma_{33}$ ) allows for a more fundamental understanding of why the shielding tensor shows this specific orientation. Fig. 3b shows the results for this analysis for the $\sigma_{11}$ component, which is the main contributor to the large deshielding of ethylene and is hence ex- 
pected to contain the most valuable information. According to the NCS analysis, the deshielding of this strongly deshielded $\sigma_{11}$ component mainly arises from a large contribution of the $\sigma(\mathrm{C}=\mathrm{C})$ orbital and smaller contributions of the $\sigma(\mathrm{C}-\mathrm{H})$ orbitals. According to Eqn. (2), a large deshielding along $\sigma_{11}$ originating from the $\sigma(\mathrm{C}=\mathrm{C})$ orbital evidences the presence of a low-lying vacant orbital, oriented perpendicular to both, $\sigma_{11}$ and the $\sigma(\mathrm{C}=\mathrm{C})$ orbital. This orbital can easily be identified as the $\pi^{*}(\mathrm{C}=\mathrm{C})$ orbital - an energetically low-lying vacant orbital. Hence, the main difference in chemical shielding between $\mathrm{sp}^{2}$ and $\mathrm{sp}^{3}$ carbons can be traced back to an orbital origin - the presence or absence of a $\pi$-system which causes a low-lying vacant orbital on the carbon atom and leads to the characteristic orientation and deshielding of the shielding tensor.

a)

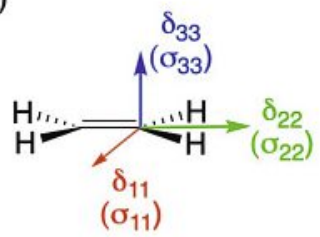

$\delta_{\text {iso }}=132 \mathrm{ppm}$

$\delta_{11}=258 \mathrm{ppm}$

$\delta_{22}=124 \mathrm{ppm}$

$\delta_{33}=14 \mathrm{ppm}$
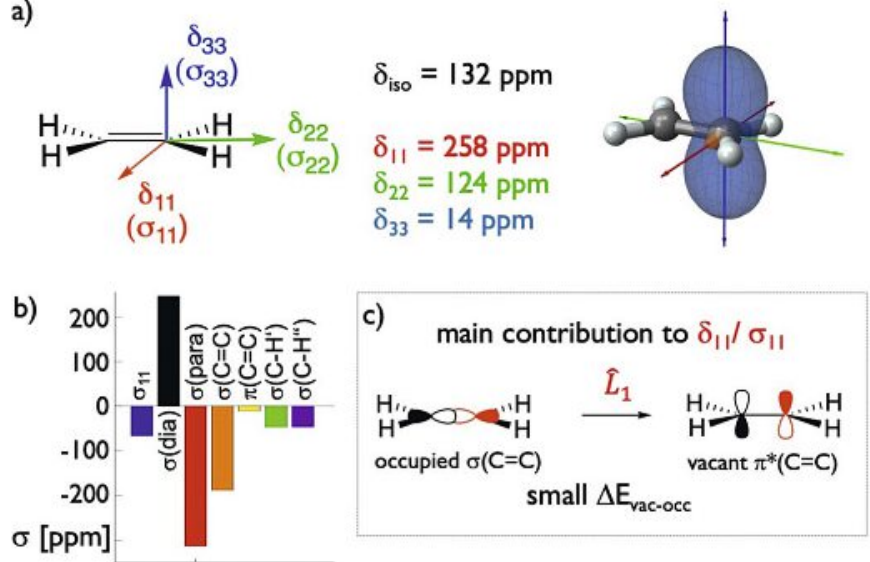

d)

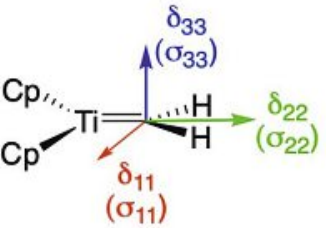

$\delta_{\text {iso }}=338 \mathrm{ppm}$

$\delta_{11}=821 \mathrm{ppm}$

$\delta_{22}=176 \mathrm{ppm}$

$\delta_{33}=18 \mathrm{ppm}$
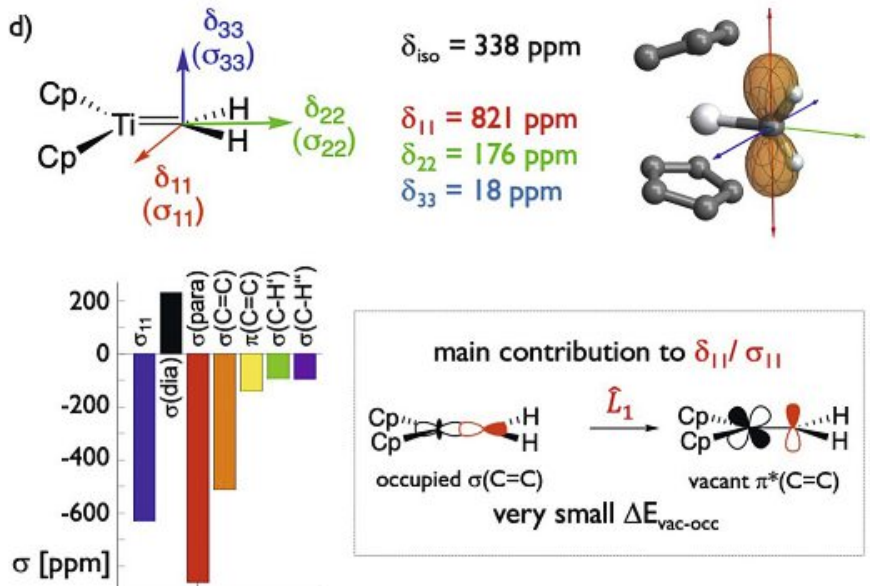

e)

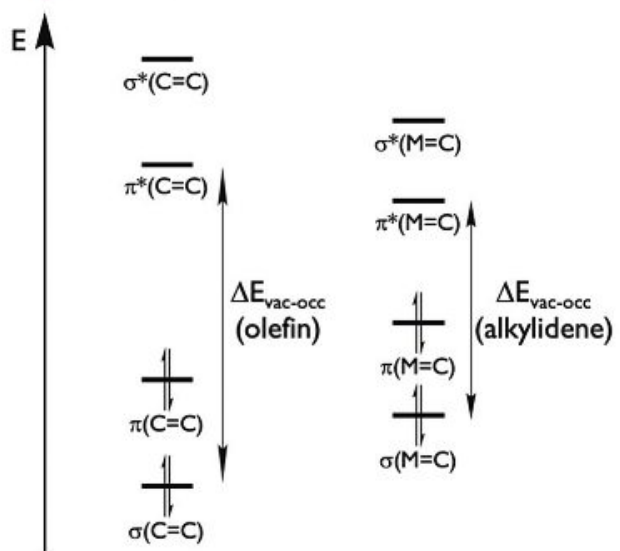

Fig. 3. a) Orientation of the chemical shielding tensor in ethylene, b) results of Natural Chemical Shielding (NCS) analysis for the principal components of the chemical shielding tensor of ethylene, and c) main orbital couplings involved in the deshielding of the $\sigma_{11}$ component of ethylene. d) Tensor orientation, NCS analysis and main orbital contributions causing deshielding in the metal alkylidene $\mathrm{Cp}_{2} \mathrm{Ti}=\mathrm{CH}_{2}$. e) Relative energy of frontier orbitals in olefins and metal alkylidenes.

\subsection{Metal Alkylidenes}

Metal alkylidenes show particularly high deshielded chemical shifts, typically around $250-350 \mathrm{ppm}$. This is somewhat perplexing, as alkylidenes typically show nucleophilic reactivity, while their chemical shift is close to that of a carbocation and far from that of a carbanion $\left(405 \mathrm{ppm}\right.$ for $\mathrm{CH}_{3}^{+}$vs. $-75 \mathrm{ppm}$ for $\mathrm{CH}_{3}^{-}$), showing that chemical shift and charge are per se unrelated properties. ${ }^{[14]}$ The orientation of the chemical shielding tensor of a prototypical carbene $\left(\mathrm{Cp}_{2} \mathrm{Ti}=\mathrm{CH}_{2}\right)$ is shown in Fig. $3 \mathrm{~d}$. Notably, the orientation of the shielding tensor in the metal alkylidene is the same as in ethylene, where one carbon fragment is replaced by a metal, albeit much more deshielded. This large deshielding is mostly due to the $\delta_{11} / \sigma_{11}$ component of the shielding tensor. A NCS analysis (Fig. 3d) of the $\sigma_{11}$ component shows that this large deshielding is mainly due to the $\sigma(\mathrm{M}-\mathrm{C})$ orbital, which indicates the presence of a low-lying vacant $\pi^{*}(\mathrm{M}=\mathrm{C})$ orbital to which it is magnetically coupled. Hence, both in ethylene and in the metal alkylidene the deshielding of the $\delta_{11} / \sigma_{11}$ component arises from the coupling of an orbital of $\sigma$-symmetry with an orbital of $\pi$-symmetry. However, the alkylidene shows a much larger deshielding, originating from a smaller energy gap between the $\sigma$ - and $\pi^{*}$-orbitals. This small energy gap is related to the high reactivity of metal alkylidenes, since they feature both a high-lying $\sigma(M-C)$ and a low-lying $\pi^{*}(M=C)$ orbital related to the presence of metal d-orbitals. ${ }^{[15]}$ In fact, the magnitude of the deshielding is characteristic of the specific type of carbenes: A comparison of nucleophilic Schrock-type alkylidenes with electrophilic Fischer-type carbenes reveals a larger deshielding of the latter, typically with $\delta_{\text {iso }}>300 \mathrm{ppm}$. This is mainly associated with a larger deshielding of the $\delta_{11} / \sigma_{11}$ component of the shielding tensor, which relates to a particularly low-lying $\pi *(\mathrm{M}=\mathrm{C})$ orbital, caused by the electron-withdrawing $\mathrm{CO}$ ligands on Fischer carbenes, hence their electrophilic nature (and not, as often implied in textbooks, related to the presence of the methoxy-substituent in the carbene ligand that in fact raises the $\pi^{*}(\mathrm{M}=\mathrm{C})$ orbital, making the carbene more stable but less electrophilic). ${ }^{[16]}$

\subsection{Metal Alkyls}

Chemical shift tensor analysis is also a useful tool to assess the electronic structure of metal alkyl compounds. A prototypical example of such a compound is the Petasis reagent, $\mathrm{Cp} \mathrm{P}_{2} \mathrm{Ti}\left(\mathrm{CH}_{3}\right)_{2}{ }^{[17]}$ While unreactive towards water, this compound is a well-known olefination agent, providing an alternative to Wittig-type chemistry. Under reaction conditions, $\mathrm{Cp}_{2} \mathrm{Ti}\left(\mathrm{CH}_{3}\right)_{2}$ undergoes $\alpha-\mathrm{H}$ abstraction and generates the alkylidene $\mathrm{Cp}_{2} \mathrm{Ti}=\mathrm{CH}_{2}$ through release of methane (Fig. 4a). ${ }^{[18]}$ This alkylidene is the active species in the olefination of carbonyl compounds. One may wonder why $\mathrm{Cp}_{2} \operatorname{Ti}\left(\mathrm{CH}_{3}\right)_{2}$ undergoes $\alpha-\mathrm{H}$ abstraction and is one of the rare di-alkyl metal complexes that engages in olefination. Noteworthy the isotropic chemical shift of the $\alpha$-carbon atom in $\mathrm{Cp}_{2} \mathrm{Ti}\left(\mathrm{CH}_{3}\right)_{2}$ is $52 \mathrm{ppm}$, which is somewhat deshielded by comparison with typical $\mathrm{sp}^{3}$ carbon atoms. A closer inspection of the shielding tensor (Fig. $4 \mathrm{~b}$ ) reveals that the deshielding of $\mathrm{Cp}_{2} \mathrm{Ti}\left(\mathrm{CH}_{3}\right)_{2}$ mainly originates from a highly deshielded $\delta_{11} / \sigma_{11}$ component $\left(\delta_{11}=118 \mathrm{ppm}\right)$ of the shielding tensor, while the other two components are rather shielded. This most deshielded $\delta_{11} / \sigma_{11}$ component is oriented perpendicular to the plane that contains the titanium atom and the two methyl-carbon atoms. An orbital analysis of this component (Fig. 4c) reveals, that the large deshielding mostly originates from a contribution of the $\sigma(\mathrm{M}-\mathrm{C})$ bonding orbital. According to Eqn. (2), a large contribution of the $\sigma(\mathrm{M}-\mathrm{C})$ bond indicates the presence of a (low-lying) vacant orbital, perpendicular to both the $\sigma(\mathrm{M}-\mathrm{C})$ bond and the direction of $\delta_{11} / \sigma_{11}$ (Fig. $\left.4 \mathrm{~d}\right)$. This orbital is of $\pi^{*}(\mathrm{M}-\mathrm{C})$ symmetry and originates from a filled p-orbital on carbon (which is also involved in the $\mathrm{C}-\mathrm{H}$ bonds), interacting with a vacant $\mathrm{d}$-orbital on 
the metal. The large deshielding of the $\delta_{11} / \sigma_{11}$ component along with its specific orientation hence points towards a $\pi$-interaction of the alpha-carbon atom with the metal - an interaction which is not obvious by looking at the Lewis structure of $\mathrm{Cp}_{2} \operatorname{Ti}\left(\mathrm{CH}_{3}\right)_{2}$. In fact, the chemical shielding tensor in $\mathrm{Cp}_{2} \mathrm{Ti}\left(\mathrm{CH}_{3}\right)_{2}$, has the same orientation as in $\mathrm{Cp}_{2} \mathrm{Ti}=\mathrm{CH}_{2}$ (Fig. 3d), providing further evidence for the presence of a double-bond character in the Petasis reagent. This analysis of the NMR chemical shift tensor of $\mathrm{Cp}_{2} \operatorname{Ti}\left(\mathrm{CH}_{3}\right)_{2}$ hence shows that this compound already carries a double bond (alkylidene) character (Fig. 4d) and is thus programmed to react via $\alpha-\mathrm{H}$ abstraction to generate $\mathrm{Cp}_{2} \mathrm{Ti}=\mathrm{CH}_{2}$ - a compound with a fully developed $\pi$-bond. ${ }^{[19]}$ Notably, the related $\mathrm{d}^{2}$ Mo-based compound $\mathrm{Cp}_{2} \mathrm{Mo}\left(\mathrm{CH}_{3}\right)_{2}$ does not show the NMR features characteristic of a double bond character in the $\mathrm{M}-\mathrm{C}$ bond. This is consistent with the absence of a vacant orbital on the metal of $\pi(\mathrm{M}-\mathrm{C})$ symmetry and concurrently the absence of reactivity towards $\alpha-\mathrm{H}$ abstraction. ${ }^{[20]} \mathrm{A}$ similar analysis has recently been used to explain the propensity of metal alkyl compounds to undergo olefin insertion ${ }^{[21]}$ and sigma-bond metathesis. ${ }^{[22]}$

a)

b)
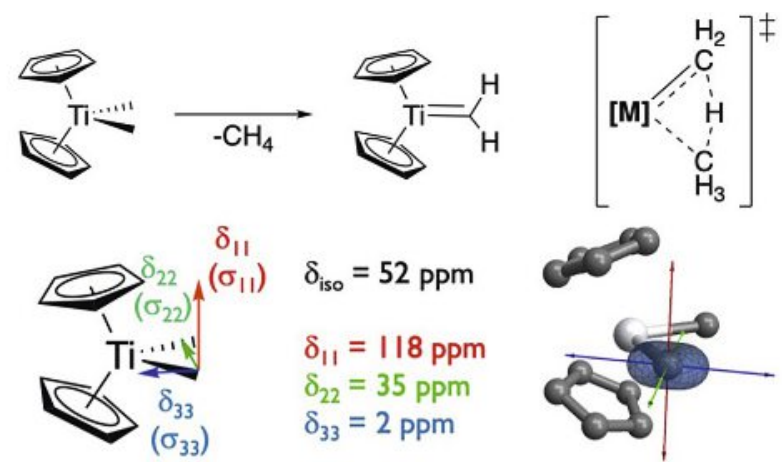

$\delta_{11}=118 \mathrm{ppm}$

$\delta_{22}=35 \mathrm{ppm}$

$\delta_{33}=2 \mathrm{ppm}$

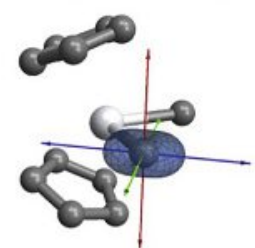

c)
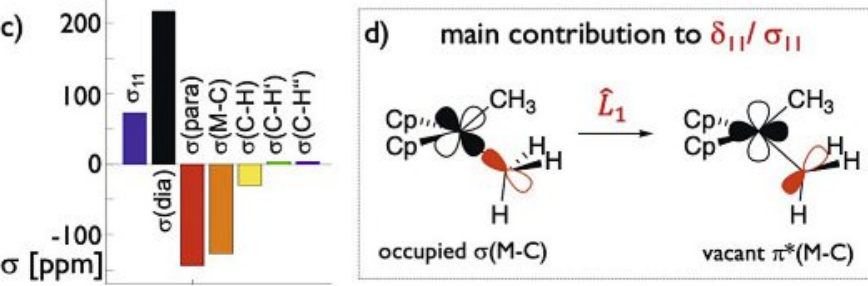

Fig. 4. a) $\alpha-\mathrm{H}$ abstraction, generating the active species in olefination by the Petasis reagent $\mathrm{Cp}_{2} \mathrm{Ti}\left(\mathrm{CH}_{3}\right)_{2}$. b) Orientation of the chemical shielding tensor in $\mathrm{Cp}_{2} \mathrm{Ti}\left(\mathrm{CH}_{3}\right)_{2}$, c) decomposition of the $\sigma_{11}$ component into individual orbital contributions (NCS analysis). d) Main orbital interaction causing the deshielding of the $\sigma_{11}$ component. Note that the large deshielding in the related alkylidene $\mathrm{Cp}_{2} \mathrm{Ti}=\mathrm{CH}_{2}$ has a similar orbital origin, showing the connection between the two compounds.

\section{Beyond ${ }^{13} \mathrm{C}$ NMR - Information Obtained from ${ }^{77} \mathrm{Se}$ and ${ }^{17} \mathrm{O}$ NMR Parameters}

The analysis of NMR parameters to obtain information on the frontier orbitals and reactivity of molecules is not limited to ${ }^{13} \mathrm{C}$ but can be expanded to other nuclei. For instance, the ${ }^{77} \mathrm{Se}$ NMR chemical shift of $[\mathrm{Se}(\mathrm{NHC})]$ adducts $(\mathrm{NHC}=N$-heterocyclic carbene) can be used to probe the electronic properties of the NHC ligand. In particular, one component of the ${ }^{77} \mathrm{Se}$ NMR chemical shift tensor (oriented along the $\mathrm{Se}=\mathrm{C}$ bond) has been shown to be highly sensitive to the substituents bound to the nitrogen atoms that influence the electronic properties. Overall, more deshielded ${ }^{77} \mathrm{Se} \mathrm{NMR}$ chemical shifts are characteristic of more $\pi$-accepting NHC ligands. This property has been used to rationalize and parametrize the selectivity of NHC-Ru-based olefin metathesis catalysts towards ethenolysis of cyclic olefins and to explain why catalysts bearing an $\mathrm{N}-\mathrm{CF}_{3}$ group outperform benchmark catalysts (Fig. 5).[23]
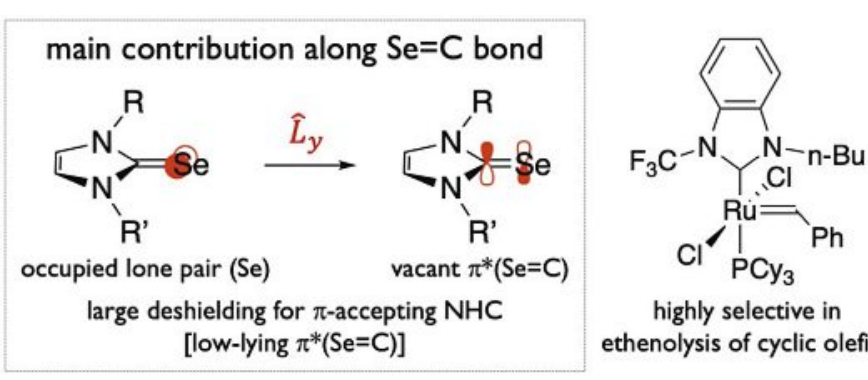

highly selective in ethenolysis of cyclic olefins

Fig. 5. Magnetically induced orbital interaction giving information on $\pi$-accepting properties of the NHC ligand and NHC-Ru based metathesis catalyst showing the highest selectivity towards ethenolysis of cyclic olefins.

Another application consists in the investigation of classical oxidants and their corresponding ${ }^{17} \mathrm{O}$ NMR parameters. Fig. 6a shows a selection of peroxide compounds: $\mathrm{H}_{2} \mathrm{O}_{2}, t \mathrm{BuOOH}$, dimethyldioxirane (DMDO), and meta-chloroperoxybenzoic acid ( $m$ CPBA). While DMDO and $m$ CPBA are active in the electrophilic epoxidation of olefins, $\mathrm{H}_{2} \mathrm{O}_{2}$ and $t \mathrm{BuOOH}$ remain unreactive unless activated by metal catalysts. The ${ }^{17} \mathrm{O}$ chemical shift value of these compounds shows a somewhat more deshielded isotropic chemical shift $\left(\delta_{\text {iso }}\right)$ in the active peroxides (DMDO and $m \mathrm{CPBA}$ ) by comparison with the unreactive species $\left(\mathrm{H}_{2} \mathrm{O}_{2}\right.$ and $\left.t \mathrm{BuOOH}\right)$. This difference is mainly due to the most deshielded $\delta_{11}$ component of the chemical shift tensor, which is much more deshielded in 'active' peroxides (Fig. 6a). Notably, in all peroxo species this $\delta_{11}$ component is oriented roughly perpendicular to the $\mathrm{O}-\mathrm{O}$ axis (Fig. 6b shows the orientation in $\mathrm{H}_{2} \mathrm{O}_{2}$ and DMDO as representative examples). An orbital (NCS) analysis of the $\delta_{11} / \sigma_{11}$ component reveals that this large deshielding is almost exclusively due to a large contribution of a lone-pair on oxygen with p-orbital symmetry (Fig. $6 c)$. The large deshielding of peroxides is caused by a coupling of this lone pair with the low-lying vacant $\sigma^{*}(\mathrm{O}-\mathrm{O})$ orbital. A particularly large deshielding, as observed for peroxides active in electrophilic epoxidation, thus points towards a high-lying lone pair on oxygen in combination with a low-lying $\sigma^{*}(\mathrm{O}-\mathrm{O})$ orbital. In fact, the strained cyclic structure in DMDO and the $\mathrm{H}$-bonding in $m$ CPBA force the two peroxo lone-pairs to be coplanar, thus raising their energy. Both the low-lying $\sigma^{*}(\mathrm{O}-\mathrm{O})$ orbital and the high-lying lone pairs favour the observed activity in electrophilic epoxidation, as these two orbitals can interact with the occupied $\pi(\mathrm{C}=\mathrm{C})$ and vacant $\pi^{*}(\mathrm{C}=\mathrm{C})$ orbitals of the olefin, respectively (Fig. 6d). The synergy of both interactions shows that in 'electrophilic' epoxidation the oxidant has both electrophilic and nucleophilic character. Similar synergistic processes can be anticipated for other 'electrophilic' additions such as halogenation of olefins by $\mathrm{X}_{2}$ or $\mathrm{NBX}(\mathrm{X}=\mathrm{Cl}, \mathrm{Br}, \mathrm{I})$. Similar considerations apply to the epoxidation of olefins by $\mathrm{H}_{2} \mathrm{O}_{2}$, catalysed by methyltrioxorhenium (MTO). In the active metal-peroxo species the peroxo lone-pairs are coplanar and their energy is further raised by the 'spectator' oxo-ligand, increasing their reactivity towards olefins. It may thus not be surprising that metal-oxo species are ubiquitous in efficient epoxidation catalysts. ${ }^{[24]}$

\section{Conclusion}

In summary, chemical shift tensors give detailed insight into the symmetry and relative energy of frontier orbitals. A close analysis of these tensors allows for understanding the electronic structure of molecules. Chemical shift is thus more than a simple number or a 'fingerprint' but it can be used as a powerful descriptor for molecular reactivity in both stoichiometric and catalytic processes such as olefin metathesis and polymerization as well as $\mathrm{C}-\mathrm{H}$ activation processes. ${ }^{[21,22,25]}$ 
a)<smiles>O</smiles><smiles>CC(C)(C)OO</smiles><smiles>CC1OO1</smiles>

$\delta_{\text {iso }}$ $195 \mathrm{ppm}$ 205 ppm

291 ppm

386 ppm

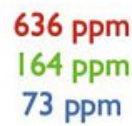

$\delta_{11} \quad 364 \mathrm{ppm}$

$\delta_{22} \quad 227 \mathrm{ppm}$

$\begin{array}{ll}\delta_{33} & -7 \mathrm{ppm}\end{array}$<smiles>O=C(OO)c1cccc(Cl)c1</smiles>

278 ppm
540 ppm

245 ppm

50 ppm
$73 \mathrm{ppm}$

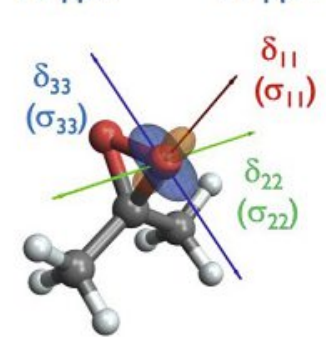

b)

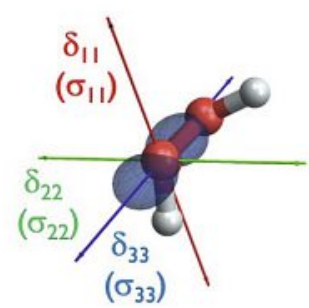

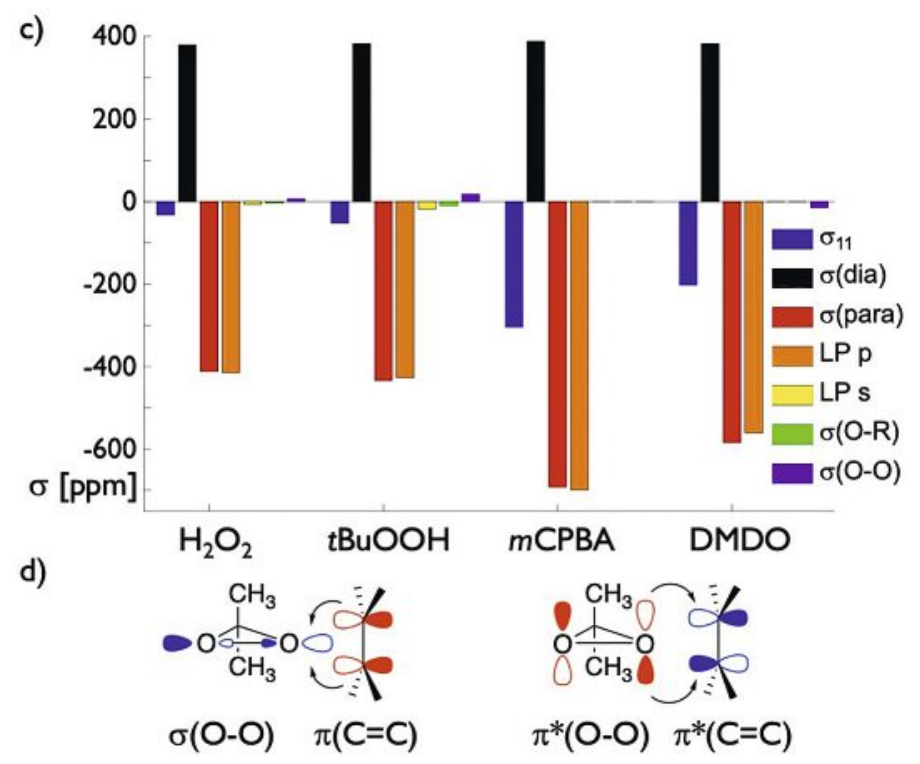

Fig. 6. a) Selected peroxo compounds and their associated chemical shift tensors (in the case of $t \mathrm{BuOOH}$ and $m \mathrm{CPBA}$ the values refer to the $\mathrm{OH}$-oxygen). b) Orientation of the shielding tensor in $\mathrm{H}_{2} \mathrm{O}_{2}$ (left) and DMDO (right). c) NCS analysis of the $\sigma_{11}$ component of the shielding tensor, and d) orbital interactions involved in 'electrophilic' epoxidation reactions.

\section{Acknowledgements}

Christopher P. Gordon is a recipient of the Scholarship Fund of the Swiss Chemical Industry (SSCI). We wish to thank Richard A. Andersen, Odile Eisenstein, Christophe Raynaud, Antonio Togni, as well as Christian Ehinger, Pascal S. Engl, Wei-Chih Liao, Keith Searles, Satoru Shirase and Keishi Yamamoto, for many discussions and their involvement in some of the original works (see references below).
[1] a) H. Günther, 'NMR Spectroscopy: Basic Principles, Concepts and Applications in Chemistry', 3 ed., Wiley-VCH, Weinheim, 2013; b) M. J. Duer, 'Introduction to Solid-State NMR Spectroscopy', Wiley-Blackwell, Hoboken, 2005; c) K. Wüthrich, Angew. Chem. Int. Ed. 2003, 42, 3340; d) D. D. Boehr, H. J. Dyson, P. E. Wright, Chem. Rev. 2006, 106, 3055.

[2] N. F. Ramsey, Phys. Rev. 1950, 78, 699.

[3] a) P. S. Pregosin, 'NMR in Organometallic Chemistry', Wiley-VCH, Weinheim, 2012; b) E. Pretsch, P. Bühlmann, M. Badertscher, 'Structure Determination of Organic Compounds', 4 ed., Springer-Verlag, Berlin Heidelberg, 2009.

[4] J. B. Lambert, R. Rittner, 'Recent advances in organic NMR spectroscopy', Norell Press, 1987.

[5] C. M. Widdifield, R. W. Schurko, Concepts Magn. Reson. Part A 2009, 34A, 91.

[6] a) J. A. Bohmann, F. Weinhold, T. C. Farrar, J. Chem. Phys. 1997, 107, 1173; b) J. Autschbach, J. Chem. Phys. 2008, 128, 164112.

[7] M. J. Frisch, G. W. Trucks, H. B. Schlegel, G. E. Scuseria, M. A. Robb, J. R. Cheeseman, G. Scalmani, V. Barone, B. Mennucci, G. A. Petersson, H. Nakatsuji, M. Caricato, X. Li, H. P. Hratchian, A. F. Izmaylov, J. Bloino, G. Zheng, J. L. Sonnenberg, M. Hada, M. Ehara, K. Toyota, R. Fukuda, J. Hasegawa, M. Ishida, T. Nakajima, Y. Honda, O. Kitao, H. Nakai, T. Vreven, J. A. Montgomery, J. E. Peralta, F. Ogliaro, M. Bearpark, J. J. Heyd, E. Brothers, K. N. Kudin, V. N. Staroverov, R. Kobayashi, J. Normand, K. Raghavachari, A. Rendell, J. C. Burant, S. S. Iyengar, J. Tomasi, M. Cossi, N. Rega, J. M. Millam, M. Klene, J. E. Knox, J. B. Cross, V. Bakken, C. Adamo, J. Jaramillo, R. Gomperts, R. E. Stratmann, O. Yazyev, A. J. Austin, R. Cammi, C. Pomelli, J. W. Ochterski, R. L. Martin, K. Morokuma, V. G. Zakrzewski, G. A. Voth, P. Salvador, J. J. Dannenberg, S. Dapprich, A. D. Daniels, Farkas, J. B. Foresman, J. V. Ortiz, J. Cioslowski, D. J. Fox, Gaussian 09 (Gaussian Inc., Wallingford CT), 2009.

[8] C. Adamo, V. Barone, J. Chem. Phys. 1999, 110, 6158.

[9] F. Jensen, J. Chem. Theory Comput. 2014, 10, 1074.

[10] a) D. Andrae, U. Häußermann, M. Dolg, H. Stoll, H. Preuß, Theor. Chim. Acta. 1990, 77, 123; b) M. Dolg, U. Wedig, H. Stoll, H. Preuss, J. Chem. Phys. 1987, 86, 866.

[11] G. te Velde, F. M. Bickelhaupt, E. J. Baerends, C. Fonseca Guerra, S. J. A. van Gisbergen, J. G. Snijders, T. Ziegler, J. Comput. Chem. 2001, 22, 931.

[12] a) E. van Lenthe, E. J. Baerends, J. G. Snijders, J. Chem. Phys. 1993, 99, 4597; b) E. van Lenthe, R. van Leeuwen, E. J. Baerends, J. G. Snijders, Int. J. Quantum Chem. 1996, 57, 281.

[13] NBO 6.0, E. D. Glendening, J. K. Badenhoop, A. E. Reed, J. E. Carpenter, J. A. Bohmann, C. M. Morales, C. R. Landis, F. Weinhold, University of Wisconsin, Madison, 2013.

[14] K. B. Wiberg, J. D. Hammer, T. A. Keith, K. Zilm, Tetrahedron. Lett. 1997, $38,323$.

[15] S. Halbert, C. Copéret, C. Raynaud, O. Eisenstein, J. Am. Chem. Soc. 2016, $138,2261$.

[16] K. Yamamoto, C. P. Gordon, W.-C. Liao, C. Copéret, C. Raynaud, O. Eisenstein, Angew. Chem. Int. Ed. 2017, 56, 10127.

[17] N. A. Petasis, E. I. Bzowej, J. Am. Chem. Soc. 1990, 112, 6392.

[18] H. van der Heijden, B. Hessen, J. Chem. Soc., Chem. Commun. 1995, 145.

[19] C. P. Gordon, K. Yamamoto, K. Searles, S. Shirase, R. A. Andersen, O. Eisenstein, C. Copéret, Chem. Sci. 2018, 9, 1912.

[20] J. C. Green, M. L. H. Green, C. P. Morley, J. Organomet. Chem. 1982, 233, C4.

[21] C. P. Gordon, S. Shirase, K. Yamamoto, R. A. Andersen, O. Eisenstein, C. Copéret, Proc. Natl. Acad. Sci. 2018, 115, E5867.

[22] C. P. Gordon, D. B. Culver, M. P. Conley, O. Eisenstein, R. A. Andersen, C. Copéret, J. Am. Chem. Soc. 2019, 141, 648 .

[23] P. S. Engl, C. B. Santiago, C. P. Gordon, W.-C. Liao, A. Fedorov, C. Copéret, M. S. Sigman, A. Togni, J. Am. Chem. Soc. 2017, 139, 13117.

[24] C. Ehinger, C. P. Gordon, C. Copéret, Chem. Sci. 2019, 10, 1786.

[25] C. P. Gordon, K. Yamamoto, W.-C. Liao, F. Allouche, R. A. Andersen, C. Copéret, C. Raynaud, O. Eisenstein, ACS Cent. Sci. 2017, 3, 759. 